\title{
A rectal cancer feasibility study with an embedded phase III trial design assessing magnetic resonance tumour regression grade (mrTRG) as a novel biomarker to stratify management by good and poor response to chemoradiotherapy (TRIGGER): study protocol for a randomised controlled trial
}

Nick J. Battersby ${ }^{1,2}$, Mit Dattani ${ }^{1}$, Sheela Rao ${ }^{3}$, David Cunningham³ ${ }^{3}$ Diana Tait ${ }^{3}$, Richard Adams ${ }^{4}$, Brendan J. Moran ${ }^{1,2}$, Shelize Khakoo ${ }^{5}$, Paris Tekkis ${ }^{6}$, Shahnawaz Rasheed ${ }^{6}$, Alex Mirnezami, Philip Quirke $^{8}$, Nicholas P. West ${ }^{8}$, Iris Nagtegaal ${ }^{9}$, Irene Chong ${ }^{10}$, Anguraj Sadanandam ${ }^{10}$, Nicola Valeri ${ }^{10}$, Karen Thomas ${ }^{11}$, Michelle Frost ${ }^{12}$ and Gina Brown ${ }^{12^{*}}$

\footnotetext{
Abstract

Background: Pre-operative chemoradiotherapy (CRT) for MRI-defined, locally advanced rectal cancer is primarily intended to reduce local recurrence rates by downstaging tumours, enabling an improved likelihood of curative resection. However, in a subset of patients complete tumour regression occurs implying that no viable tumour is present within the surgical specimen. This raises the possibility that surgery may have been avoided. It is also recognised that response to CRT is a key determinant of prognosis. Recent radiological advances enable this response to be assessed pre-operatively using the MRI tumour regression grade ( $\mathrm{mrTRG).} \mathrm{Potentially,} \mathrm{this} \mathrm{allows}$ modification of the baseline MRI-derived treatment strategy. Hence, in a 'good' mrTRG responder, with little or no evidence of tumour, surgery may be deferred. Conversely, a 'poor response' identifies an adverse prognostic group which may benefit from additional pre-operative therapy.

(Continued on next page)
}

\footnotetext{
* Correspondence: gina.brown@rmh.nhs.uk

${ }^{12}$ Department of Radiology, Royal Marsden Hospital Sutton, Sutton SM2 5PT, UK

Full list of author information is available at the end of the article
} International License (http://creativecommons.org/licenses/by/4.0/), which permits unrestricted use, distribution, and reproduction in any medium, provided you give appropriate credit to the original author(s) and the source, provide a link to the Creative Commons license, and indicate if changes were made. The Creative Commons Public Domain Dedication waiver (http://creativecommons.org/publicdomain/zero/1.0/) applies to the data made available in this article, unless otherwise stated. 
(Continued from previous page)

Methods/design: TRIGGER is a multicentre, open, interventional, randomised control feasibility study with an embedded phase III design. Patients with MRI-defined, locally advanced rectal adenocarcinoma deemed to require CRT will be eligible for recruitment. During CRT, patients will be randomised (1:2) between conventional management, according to baseline MRI, versus mrTRG-directed management. The primary endpoint of the feasibility phase is to assess the rate of patient recruitment and randomisation. Secondary endpoints include the rate of unit recruitment, acute drug toxicity, reproducibility of mrTRG reporting, surgical morbidity, pathological circumferential resection margin involvement, pathology regression grade, residual tumour cell density and surgical/specimen quality rates. The phase III trial will focus on long-term safety, regrowth rates, oncological survival analysis, quality of life and health economics analysis.

Discussion: The TRIGGER trial aims to determine whether patients with locally advanced rectal cancer can be recruited and subsequently randomised into a control trial that offers MRI-directed patient management according to radiological response to CRT (mrTRG). The feasibility study will inform a phase III trial design investigating stratified treatment of good and poor responders according to 3-year disease-free survival, colostomy-free survival as well as an increase in cases managed without a major resection.

Trial registration: ClinicalTrials.gov, ID: NCT02704520. Registered on 5 February 2016.

Keywords: Randomised control trial, Chemoradiotherapy, Rectal cancer, mrTRG, Complete response, Tumour regression, Tumour cell density

\section{Background}

Currently, $45-55 \%$ of rectal cancer patients receive pre-operative chemoradiotherapy (CRT) for locally advanced disease at presentation. Pre-operative CRT is given in order to downstage the tumour. It has three potential benefits in high-risk rectal cancers that respond to treatment: an increased likelihood of a clear circumferential resection margin (CRM); less radical surgery than was initially planned on the baseline staging magnetic resonance imaging (MRI) [1]; and in a small subset of patients in whom there is no clinical or radiological evidence of tumour, the option of deferral of surgery [2, 3]. However, the response to treatment is highly variable, with up to $30 \%$ of patients achieving a complete or near-complete response as judged by pathological tumour regression grade (pTRG). Prognostically, a good pTRG is associated with significantly lower local recurrence, distant metastatic rates and with higher overall survival compared with a poor PTRG $[4,5]$.

The need for a validated means of assessing response to treatment is widely accepted [6] but there has been no reliable method of assessing this response in the pre-operative setting to date. Therefore, the current consensus is that the baseline MRI staging should be the standard of care that is used to define the plane of surgery, regardless of any assessment of treatment response [7]. Recently, a 5-point MRI tumour regression grade (mrTRG), which most closely resembles the Mandard pTRG system [8], has been developed [9]. The basic principle of both grading systems relates to the ratio of tumour to fibrosis following CRT (Table 1). Patients with a poor CRT response have a 5 -year overall survival of $27 \%$ versus $72 \%(p=0.001)$ for a good CRT response [10]. This novel imaging biomarker has been reliable and reproducible between multiple independent radiologists, and validated against both pathology and survival outcomes [10-12]. However, there is currently insufficient evidence that this information can be safely used to alter treatment decisions.

\section{The 'good-response' group (mrTRG I and II)}

The complications of surgery can be considerable. The 90-day mortality rate from a major bowel resection is $3-6 \%$ [13], significant morbidity is frequent and long-term functional impairment is common, even in the presence of restorative surgery [14-16]. When a pathological complete response (pCR) to CRT occurs, with no residual cancer identified, this raises the possibility that the risks of surgery may have been avoided by watchful waiting or deferral of surgery [17]. Pioneered by Professor Angelita HabrGama, several cohorts have demonstrated this to be feasible and safe; avoiding radical surgery by close surveillance of selected patients in whom there is clinical disappearance of the tumour following CRT

\footnotetext{
Table 1 Magnetic resonance imaging tumour regression grade (mrTRG)

mrTRG 1 - Complete radiological response (linear scar only) mrTRG 2 - Good response (dense fibrosis, no obvious tumour signal) mrTRG 3 - Moderate response ( $>50 \%$ fibrosis and visible intermediate signal) mrTRG 4 - Slight response (mostly tumour)

mrTRG 5 - No response/regrowth of tumour
} 
$[2,3,18]$. Termed as a clinical complete response (cCR), the combination of digital rectal examination, endoscopy and imaging are used to define and monitor patients under a deferral of surgery protocol as a surrogate for a $\mathrm{pCR}$, thereby avoiding radical surgery [19-21]. However, current techniques for assessing a cCR are unreliable, and up to two thirds of patients are not identified pre-operatively [22-25]. By using the novel mrTRG biomarker prospectively in a randomised controlled study, it may be possible to determine the precise safety of this approach, whilst increasing the proportion of $\mathrm{CCR}$ patients identified using an objective and validated tool.

A good tumour response on post-CRT MRI (mrTRG I and II) may enable a nonoperative approach to rectal cancer. Consequently, patients may have a reduced overall morbidity and mortality [26], as well as an improvement in quality of life (QoL).

\section{The 'poor-response' group (mrTRG III-V)}

The TRIGGER trial will offer poor responders consolidation therapy using a fluorouracil (5-FU)-based regimen combined with oxaliplatin (infusional 5-FU or capecitabine with oxaliplatin, FOLFOX or CAPOX). These regimens have been shown to be effective systemic treatments for colorectal malignancy and they are recommended by most international guidelines $[27,28]$, usually in the post-operative setting. However, a prospective study by Garcia-Aguilar et al. found that FOLFOX can be used as preoperative consolidation therapy, along with conventional CRT, without evidence of additional toxicity [29]. In the SOCRATES trial, patients received a similar regimen of radiotherapy and oxaliplatin; however, capecitabine (the 5-FU oral prodrug) was used [30]. The CAPOX treatment regime was offered to patients with locally advanced rectal cancer, 82 of the 83 patients enrolled into the trial completed the preoperative CAPOX regimen and 78 patients proceeded to surgery. Similar compliance rates were seen with pre-operative CAPOX-RT in a German study, where compliance rates were 96\% [31]. In TRIGGER, 'poor responders' (mrTRG III-V) will be offered systemic oxaliplatin and either 5-FU or capecitabine pre-operatively. In this high-risk subgroup of patients, the earlier introduction of systemic therapy appears not only to improve compliance but also tumour response rates and the rates of distant metastatic disease [32].

\section{Methods/design}

\section{Trial overview}

The TRIGGER trial is a multicentre, open, randomised control trial (Additional file 1). Randomisation is 2:1 in favour of mrTRG-directed management. We anticipate that 30 centres will recruit to the study. The phase III primary endpoints will compare outcomes by intentionto-treat analysis between the control arm and the interventional arm. There are two prospective subtrials, the first involves the 'good-response' group and the second involves the 'poor-response' group. The trial flow chart is shown in Fig. 1 (Additional files 2 and 3). The trial has been developed in accordance with Standard Protocol Items: Recommendations for Interventional Trials (SPIRIT) guidelines (Additional file 4).

\section{Study population and eligibility criteria}

Eligible patients are 18 years or older at the time of diagnosis, able to give informed consent, and have a biopsy-confirmed adenocarcinoma $0-15 \mathrm{~cm}$ from the anal verge measured on MRI or rigid sigmoidoscopy. The initial staging MRI must indicate locally advanced rectal carcinoma, defined here as: mrCRM unsafe, $\geq \mathrm{mrT3c}(>5 \mathrm{~mm}$ beyond muscularis propria), mrN1c (extramural tumour deposit not typical of lymph nodes) or MRI-assessed evidence of extramural venous invasion (mrEMVI)-positive disease (extramural venous invasion on MRI). The patient must be deemed to require chemoradiotherapy and scheduled to receive 45 Gy-55 Gy long-course radiotherapy.

Patients will be ineligible if there is evidence of distant metastatic disease, including resectable liver metastases; MRI contraindications; intolerance or contraindication to planned chemoradiotherapy (CRT); receive alternative cytotoxic or investigational drug treatment outside of protocol stipulation; pregnancy; breastfeeding; other malignant disease within the preceding 5 years with the exception of nonmelanomatous skin cancer, carcinoma in situ and early stage disease with $<5 \%$ recurrence risk. Some centres report the controversial practice of offering CRT to early stage rectal cancer patients with the specific intention of avoiding an APE by achieving a cCR [20]. Only patients with locally advanced disease are eligible for TRIGGER because the multi-European centre low rectal cancer study (MERCURY II) found that patients with early stage low rectal cancer (cancer that did not breach the CRM or low rectal plane on MRI) were able to undergo a restorative resection the majority of the time $(108 / 166$ - 65\%) [33]. Without radiotherapy this group reported a pCRM rate of $2 \%$ and these patients avoided the morbidity and functional impairment that is associated with radiotherapy.

This is a multicentre study and currently seven trials units are open to recruitment; we anticipate this will be sufficient to deliver the feasibility study. A further 20 centres are undergoing the trials registration and quality assurance process with a view to participating in 


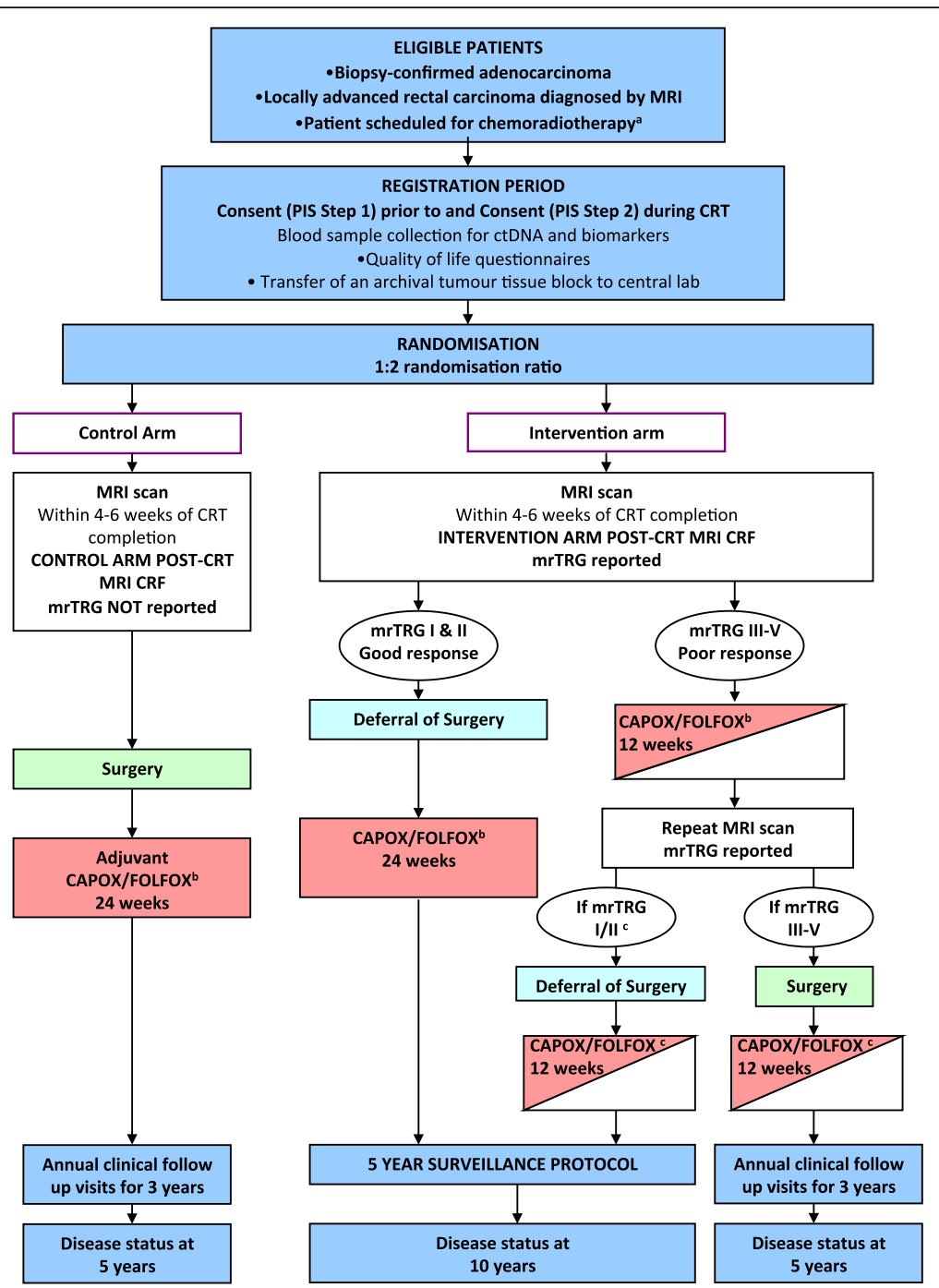

Fig. 1 a Scheduled to receive 45 Gy-55 Gy long-course radiotherapy. b Treatment decision should be made prior to registration (planned choice is a randomisation stratification variable). Medical oncologist may choose to use CAPOX or FOLFOX, or single-agent capecitabine or 5-FU if concomitant use of oxaliplatin is contraindicated. c Patient defers surgery then the remaining 12 weeks of chemotherapy should be given as soon as possible following the repeat magnetic resonance imaging (MRI) scan and multidisciplinary team (MDT) meeting

feasibility and/or the phase III trial. Participating centres must have an established multidisciplinary team (MDT) that includes a minimum of one oncologist, one histopathologist, one radiologist and one surgeon, and be able to accommodate and deliver all aspects of the protocol, including patient follow-up, particularly the follow-up requirements for safe deferral of surgery. The centre is required to meet eligibility criteria and trial conduct must comply with the protocol as agreed by the sponsor, the Medicines and Healthcare products Regulatory Agency (MHRA) and the Multicentre Ethical Committee (MREC). Trial centres will encompass both teaching and district general hospitals to enhance generalisability.

\section{Study objectives \\ Feasibility study objectives}

- Patient recruitment - the number of eligible patients willing to enter the study, and the rate of concordance with the allocated treatment plan

- Centre recruitment - acceptability of the trial concept and the protocol to each unit as well as the ability of radiologists to reliably assess response (agreement with central radiologist measured using a training set with kappa $>0.7$ )

- Evaluate the reproducibility of mrTRG by assessing the strength of the agreement between the recruiting radiologists and the central radiologist 
- Assess the clinical, radiological response rate in the control versus intervention arm. Pathological response rate will also be reported and compared when possible

- Evaluate safety by assessing acute drug toxicity and 30-day surgical morbidity

- Compare the control arm versus the intervention arm in terms of pCRM involvement and specimen quality as a proxy for quality of surgery (quality graded by plane of surgery as: complete clinical response (no specimen), mesorectal, intramesorectal, and muscularis propria). The assessment will include patients who undergo surgery for tumour regrowth following initial management with deferral of surgery

\section{Phase III study objectives}

- The primary objective of the phase III trial will be to compare 3-year disease-free survival (DFS) in the control arm versus the mrTRG-directed management arm

- Overall survival (OS), colostomy-free survival (CFS), distant (DR) and local recurrence (LR) in the control arm versus the mrTRG-directed management arm, and tumour regrowth rates in patients treated with deferral of surgery

- QoL comparison

- Cost-utility analysis

\section{Sample size}

The feasibility study is designed to determine whether proceeding to a phase III study is realistic. Using the phase III primary endpoint of 3-year DFS, an expected improvement in DFS for the intervention group by intention-to-treat analysis would be $82 \%$ from $74 \%$ (i.e. a hazard ratio of 0.66 ) with $80 \%$ power and a $5 \%$ two-sided level of statistical significance. This would require 633 patients: 422 in the intervention arm and 211 in the control arm based on a 2:1 allocation ratio to be recruited over 3 to 5 years with at least 3 years' follow-up. Hence, in order for the phase III trial to be viable, recruitment rates of at least 5-6 per month (to recruit 633 patients in 5 years) and, ideally, 11 per month (to recruit 633 patients in 3 years) should be achievable. The feasibility study was, therefore, designed to test the null hypothesis that the maximum possible recruitment rate is no more than 6 per month, and power calculations were performed under the assumption that the a rate of 11 per month can be achieved. The type-I error significance threshold (alpha) was set at 0.1, with a type-II error $(\beta)$ at $5 \%$ with the resulting power $(1-\beta)$ at $95 \%$ (calculated using simulation). In order to allow for a set-up period of 6 months we will recruit for a total of 10 months, with recruitment rates for the last 4 months used to test the primary hypothesis. Thus, during the final 4 months recruitment is expected to be 11 patients per month and can be no less than 6 patients per month ( 6 per month results in 5.5 years total recruitment time).

\section{Randomisation}

Patients identified at the MDT as potentially eligible for TRIGGER are invited and may consent to register prior to CRT. Basic demographic and clinical information will be collected. During CRT, eligible patients are then considered for randomisation. Registered patients who are not randomised are retained along with the reason for nonrandomisation, so that the causes for 'drop-out' and estimates of generalisability can be established.

Randomisation is performed centrally, at the TRIGGER trial office, by a computer-based randomisation algorithm. Patients are randomised 2:1 in favour of mrTRG-directed management (interventional arm) over best current management (control arm) based on the index staging MRI. To avoid chance imbalance in the two arms, randomisation will factor-in stratification variables: recruiting site, mrEMVI status, tumour height, and planned choice of systemic chemotherapy.

Following randomisation, the radiologist from the recruiting centre will report the mrTRG for the intervention arm but not for the control arm. It is not possible to blind the patient, radiologist, surgeon or pathologist, but these assigned members of the MDT will be trained to manage patients according to the TRIGGER protocol (v5.0) and to prospectively proforma report findings. To further enhance the quality, reliability and reproducibility of the data the radiologists and pathologists will centrally review image quality, surgical specimen quality and pathological tumour regression.

\section{Intervention}

The intervention in this study results from mrTRGdirected management. To report the mrTRG, the baseline and post-treatment MRI scans will be reported according to standardised proformas and the following imaging standards are required:

- Positioning and patient preparation - buscopan 20 $\mathrm{mg}$ (intra-muscularly) to be given, or other suitable antispasmodic agents according to local protocol. Superior saturation bands/REST slabs for adequate abdominal motion suppression and anterior SAT band to be used in conjunction with AP-phase encoding direction to reduce image degradation due to abdominal wall motion. Firm surface coil placement - lower edge of coil $10 \mathrm{~cm}$ below symphysis pubis 
Table 2 Assessment schedule summary: control arm

\begin{tabular}{|c|c|c|c|c|c|c|c|c|c|c|c|c|}
\hline \multirow{2}{*}{$\begin{array}{l}\text { Control arm } \\
\text { Visit type }\end{array}$} & \multirow[b]{2}{*}{$\begin{array}{l}\text { Prior to } \\
\text { patient } \\
\text { entry }\end{array}$} & \multirow{2}{*}{$\begin{array}{l}\begin{array}{l}\text { Registration } \\
\text { period }\end{array} \\
\text { Registration }\end{array}$} & \multicolumn{6}{|c|}{ Intervention phase } & \multicolumn{3}{|c|}{$\begin{array}{l}\text { Annual } \\
\text { follow-up }\end{array}$} & \multirow{2}{*}{$\begin{array}{l}\begin{array}{l}\text { Disease } \\
\text { status }^{9}\end{array} \\
60\end{array}$} \\
\hline & & & $\begin{array}{l}\text { Randomisation } \\
\text { (baseline) }\end{array}$ & $\begin{array}{l}\text { Post } \\
\text { CRT }\end{array}$ & MDT & surgery & $\begin{array}{l}\text { Surgical } \\
\text { follow-up }\end{array}$ & $\begin{array}{l}\text { Adjuvant chemotherapy for } \\
24 \text { weeks }\end{array}$ & 12 & 24 & 36 & \\
\hline Timelines & & $\begin{array}{l}\leq 4 \text { weeks } \\
\text { prior to } C R T\end{array}$ & During CRT & $\begin{array}{l}4-6 \\
\text { weeks } \\
\text { post } \\
\text { CRT }\end{array}$ & & $\begin{array}{l}6-12 \\
\text { weeks } \\
\text { post CRT }\end{array}$ & $\begin{array}{l}6 \text { weeks } \\
\text { post } \\
\text { surgery }\end{array}$ & $\begin{array}{l}\text { Toxicity assessed at } \\
\text { end of each chemotherapy } \\
\text { cycle }\end{array}$ & & $\begin{array}{l}\text { nths } \\
\text { CRT }\end{array}$ & from & end \\
\hline Informed consent ${ }^{a}$ & & $x$ & $x$ & & & & & & & & & \\
\hline $\begin{array}{l}\text { Check eligibility } \\
\text { criteria }\end{array}$ & & $x$ & $x$ & & & & & & & & & \\
\hline $\begin{array}{l}\text { Diagnosis, history } \\
\text { and clinical } \\
\text { assessment }\end{array}$ & & $x$ & & & & & & & & & & \\
\hline Randomisation & & & $x$ & & & & & & & & & \\
\hline Quality of life & & $x$ & & & & & & & $x$ & & $x$ & \\
\hline Chemoradiotherapy & & & $x$ & & & & & & & & & \\
\hline Blood sample ${ }^{b}$ & & $x$ & & $x$ & & & $x$ & & $x$ & $x$ & $x$ & \\
\hline Baseline MRI & $x$ & & & & & & & & & & & \\
\hline Restaging $\mathrm{MRI}^{\mathrm{C}}$ & & & & $x$ & & & & & & & & \\
\hline Surgery & & & & & & $x$ & & & & & & \\
\hline Surgical morbidity ${ }^{f}$ & & & & & & & $x$ & & $x$ & & & \\
\hline Pathology ${ }^{d}$ & & & & & & & $x$ & & & & & \\
\hline Chemotherapy & & & & & & & & $X$ end of each cycle & & & & \\
\hline Toxicity assessment & & & & & & & & $X$ end of each cycle & $x$ & & & \\
\hline Annual follow-up & & & & & & $x$ & & & $x$ & $x$ & $x$ & $x$ \\
\hline Adverse events ${ }^{\mathrm{e}}$ & & & & $x$ & & $x$ & $x$ & $X$ end of each cycle & & & & \\
\hline $\begin{array}{l}\text { Concurrent } \\
\text { medications }\end{array}$ & & $x$ & $x$ & $x$ & & $x$ & $x$ & $X$ end of each cycle & & & & \\
\hline
\end{tabular}

$C R T$ pre-operative chemoradiotherapy, MDT multidisciplinary team, MRI magnetic resonance imaging

The $\mathrm{X}$ also denotes that Clinical Report Forms (CRFs) need completing

${ }^{a}$ Eligible subjects will be asked to provide written informed consent at registration and before randomisation

${ }^{\mathrm{b}}$ If patient has consented to additional blood sample collection for research

'The post-CRT MRI should to be performed within 4-6 weeks (maximum of 10 weeks) from completion of CRT

${ }^{\mathrm{d}}$ Resected specimen will be prepared and evaluated using a standardised protocol

${ }^{\mathrm{e}}$ All adverse events will be recorded from the date the post-CRT MRI scan is performed until 30 days after the last dose of chemotherapy is administered during the intervention phase of the trial

fBoth early (4-6 weeks) and late surgical complications (at 12 months) will be recorded

${ }^{9}$ Disease status at 5 years (does not require clinic visit): alive without metastatic or recurrent disease, alive with metastatic and/or recurrent disease (date diagnosed), dead (date of death)

- $\mathrm{T} 2$-weighted images - TR $>3500 \mathrm{~ms}$, $\mathrm{TE}>80 \mathrm{~ms}$

- Adequate signal to noise - at least four acquisitions and 6 min per sequence

- Adequately high resolution scans - field of view and matrix parameters should not exceed a pixel size of $0.6 \mathrm{~mm} \times 0.6 \mathrm{~mm}$; either $200 \mathrm{~mm} \times 200 \mathrm{~mm}$ with $384 \times 384$ matrix or $160 \mathrm{~mm} \times 160 \mathrm{~mm}$ with a $256 \times$ matrix. Slice-thickness $3 \mathrm{~mm}$ giving a 0.6 $\mathrm{mm} \times 0.6 \mathrm{~mm} \times 3 \mathrm{~mm}=1.1-\mathrm{mm}^{3}$ voxel

- Adequate coverage - high-resolution scans extend to at least $5 \mathrm{~cm}$ above the top of tumour. Any discontinuous deposits seen on sagittal view are also covered on the high resolution axial views
The post-CRT MRI should be performed within 4-6 weeks, and no later than 10 weeks of CRT completion.

\section{Control Arm}

The flow chart for the control group is shown in Fig. 1. The mrTRG is not reported for the control arm and surgery is performed within 6-12 weeks from CRT completion according to the baseline MRI staging. The follow-up and Clinical Report Form (CRF) assessment schedule is summarised in Table 2. 
Experimental intervention - good response (mrTRG I and II) The flow chart for the good-response group of the intervention arm is shown in Fig. 1. This group has been randomised to mrTRG-directed management and, where the post-treatment MRI suggests a good response (mrTRG I or II), the option of deferral of surgery is discussed with the patient and intensive active monitoring is undertaken. This will involve a combination of clinical assessments, imaging surveillance, and endoscopy at regular intervals, as per the schedule shown in Table 3 . These patients will subsequently receive 24 weeks of chemotherapy (eight cycles of CAPOX or 12 cycles of FOLFOX) and this should start within 12 weeks of CRT completion. The follow-up and CRF assessment schedule is summarised in Table 4.

\section{Detection of local regrowth}

A regrowth is a defined as recorded evidence of disease at the site of the primary tumour in a patient with previous investigations suggesting an apparent cCR. Patients with evidence of clinical or radiological local regrowth or pelvic relapse must be treated at least as urgently as a primary rectal cancer by the surgical team (Fig. 2). A biopsy identifying regrowth of adenocarcinoma should ideally be sought before planning surgical intervention and, in equivocal cases, a MDT discussion should take place to decide on the best course of action. If a patient refuses, or is not fit for, surgery then a 'Discontinuation of Trial Treatment Form' should be completed and the patient followed up unless they withdraw consent to further data collection. Follow-up will also monitor for unsalvageable regrowth - a tumour with radiological evidence of a good response that progresses following initial deferral of surgery management. The extent of progression means that the patient develops inoperable disease or $p C R M$ involvement.

Experimental intervention - poor response (mrTRG III-V) The flow chart for the poor response group of the intervention arm is shown in Fig. 1. This group has been randomised to mrTRG-directed management and the post-treatment MRI suggests a poor response (mrTRG III-V). The option of intensified additional chemotherapy is discussed with the patient, and if the patient consents, 12 weeks of chemotherapy will be given (four cycles of CAPOX or six cycles of FOLFOX); this should start within 12 weeks of CRT completion. Following the completion of 12 weeks of chemotherapy, a repeat MRI should be performed 46 weeks later and mrTRG reported again. If this MRI suggests a good response (mrTRG I or II), deferral of surgery can be discussed; otherwise, the patient

Table 3 Deferral of surgery follow-up protocol

\begin{tabular}{|c|c|c|c|c|c|}
\hline Time line from end of CRT & Visit window & Clinic $\mathrm{OPA}^{\mathrm{b}}$ & $\mathrm{PROM}^{\mathrm{C}}$ & Scans & Endoscopy \\
\hline 6 months $^{\mathrm{a}, \mathrm{d}}$ & \pm 1 month & $x$ & & MRI & Flex sig \\
\hline 9 months $^{d}$ & \pm 1 month & $x$ & & MRI & Flex sig \\
\hline 1 year $^{d}$ & \pm 1 month & $x$ & EORTC QLQ-C30, LARS, EQ-5D & $\begin{array}{l}\text { MRI } \\
\text { CT }\end{array}$ & Colonoscopy \\
\hline 1 year 3 months & \pm 1 month & $x$ & & & \\
\hline 1 year 6 months $^{d}$ & \pm 1 month & $x$ & & MRI & Flex sig \\
\hline 1 year 9 months & \pm 1 month & $x$ & & & \\
\hline 2 years $^{e}$ & \pm 1 month & $x$ & & $\begin{array}{l}\text { MRI } \\
C T\end{array}$ & Flex sig \\
\hline 2 years 6 months $^{e}$ & \pm 1 month & $x$ & & & \\
\hline 3 years $^{d}$ & \pm 2 months & $x$ & EORTC QLQ-C30, LARS, EQ-5D & $\begin{array}{l}\text { MRI } \\
C T\end{array}$ & Flex sig \\
\hline 3 years 6 months & \pm 2 months & $x$ & & & \\
\hline 4 years $^{d}$ & \pm 2 months & $x$ & & MRI & Flex sig \\
\hline 4 years 6 months & \pm 2 months & $x$ & & & \\
\hline 5 years $^{d}$ & \pm 3 months & $x$ & EORTC QLQ-C30, LARS, EQ-5D & MRI & Colonoscopy \\
\hline
\end{tabular}

${ }^{a}$ This visit should take place once the patient has completed chemotherapy. It is recommended that a computed tomography (CT) scan is also performed following the completion of chemotherapy as is usual practice

${ }^{\mathrm{b}}$ Each clinic outpatient appointment (OPA) should include a digital rectal exam and CEA (tumour marker) blood test

'Quality of life Case Record Form (CRF): EORTC QLQ-C30, European Organisation for Research and Treatment of Cancer Quality of Life Questionnaire-Core 30, v3; LARS, Low Anterior Resection Syndrome Score; EQ-5D, EuroQol Group five dimensions Health Questionnaire

If patient has consented to the additional blood sample collection for research (circulating tumour DNA and markers of cell proliferation and apoptosis) then samples collected during the clinic outpatient appointment, ideally at the same time as the routine blood tests are performed

CRT pre-operative chemoradiotherapy, Flex sig flexible sigmoidoscopy, PROM patient-reported outcome measure 


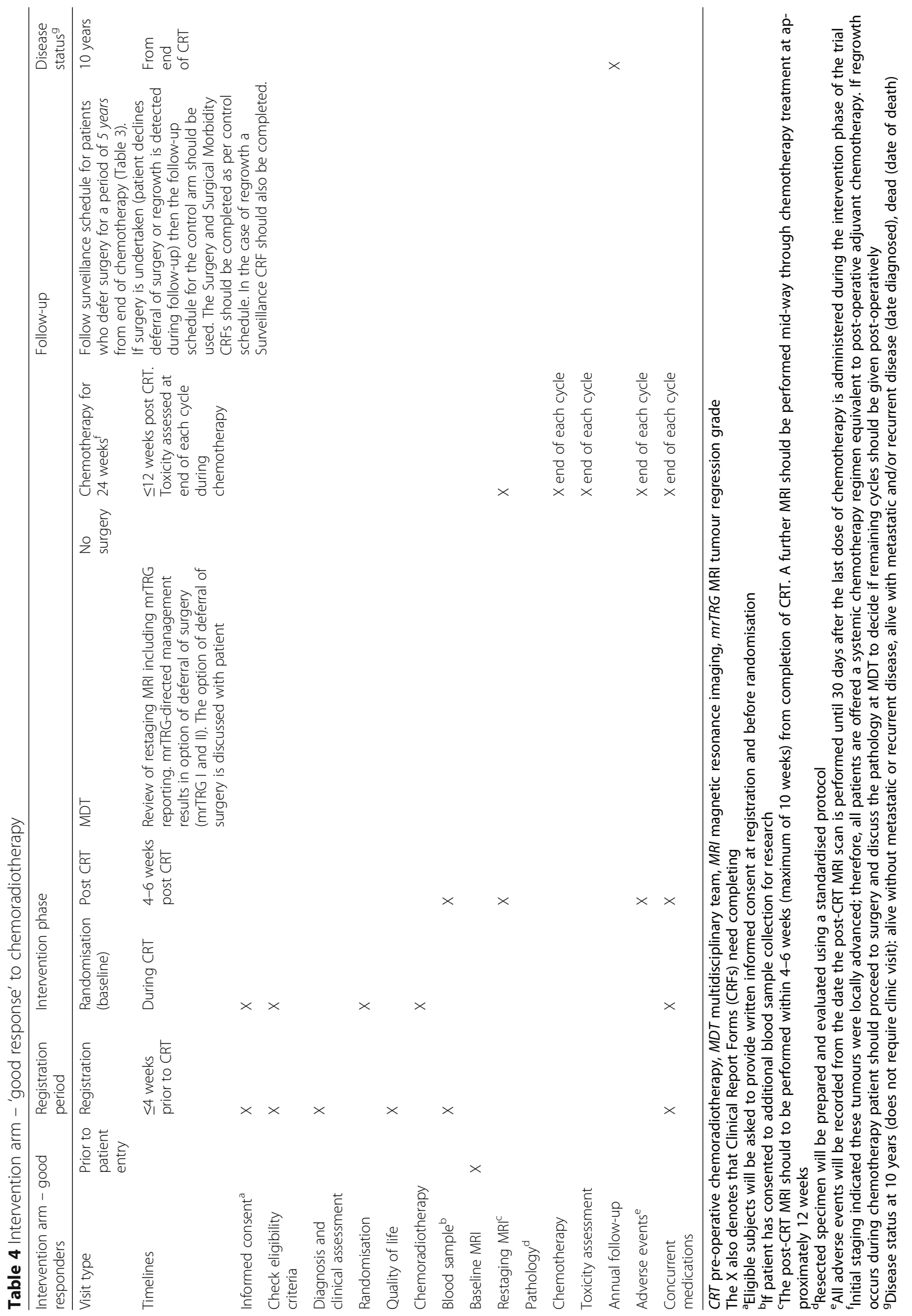




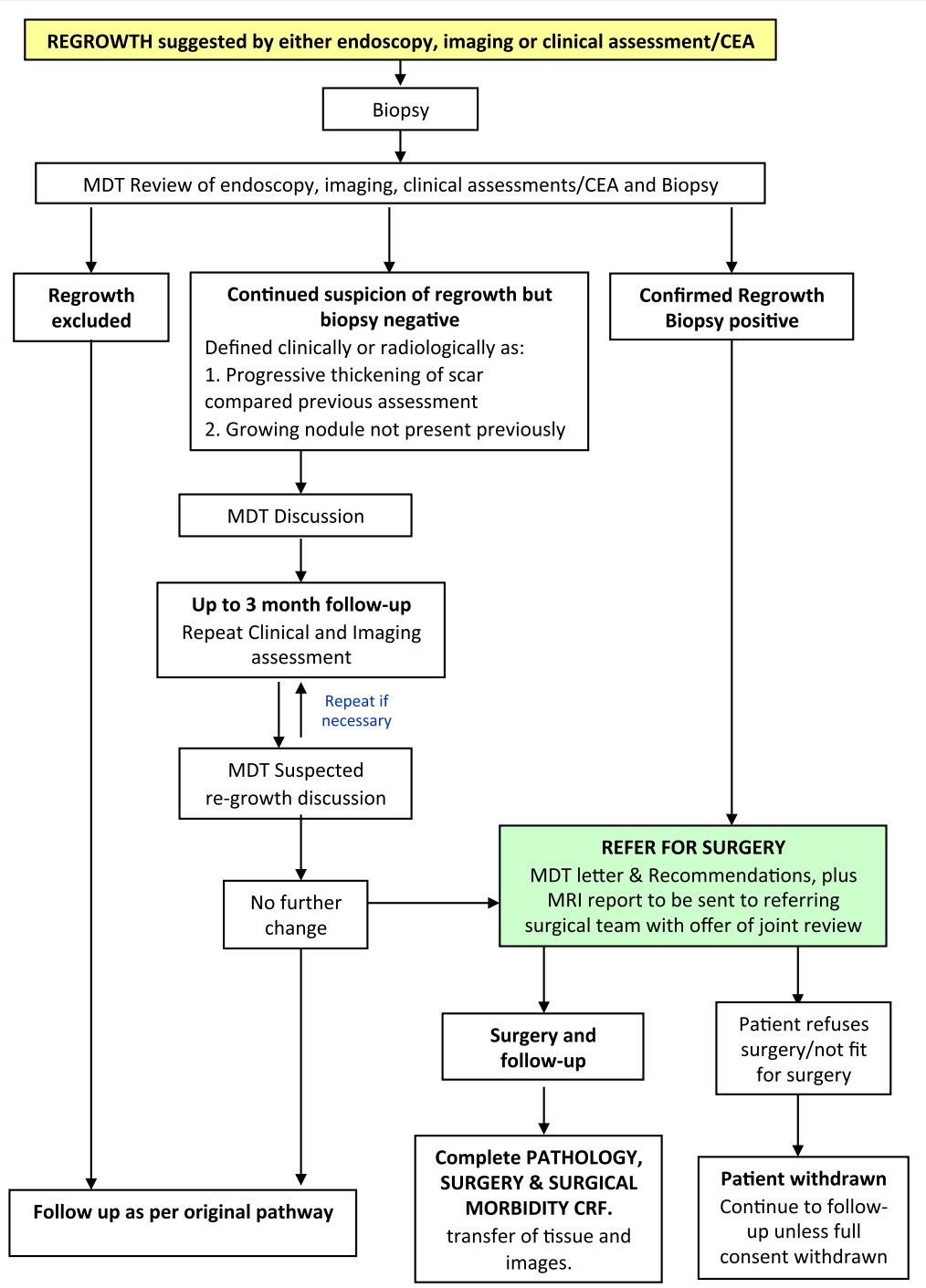

Fig. 2 Flow chart for the management of tumour regrowth after initial deferral of surgery

should proceed to surgery according to the baseline and post-treatment imaging. The follow-up and CRF assessment schedule is summarised in Table 5. The SPIRIT diagram for both randomisation arms and intervention arm sub-groups is shown in Table 6.

\section{Translational studies}

The molecular determinants of response to CRT in rectal cancer are currently poorly understood. The TRIGGER trial represents a unique opportunity to undertake comprehensive 'genotype-phenotype' comparisons as this will enable validation studies for candidate biomarkers from preliminary studies [34]. Patients will be asked to consent to the transfer of their tumour and normal mucosal tissue from the pre-treatment biopsy and post-treatment resection specimen to the central laboratory (Institute of
Cancer Research/Royal Marsden BRC, UK), and the collection of an additional research blood samples. Slides will be assessed for pathological tumour regression and tumour cell density centrally (Leeds Institute of Cancer and Pathology, UK) and tissue will also be assessed in collaborating centres (Radboud University Nijmegen, Netherlands and University of Southampton, UK).

\section{Patient-reported outcome measures (PROMs)}

The QoL aspect will be evaluated through validated self-administered PROMs questionnaires at 12 and 36 months post completion of CRT. All patients will be requested to fill out a European Organisation for Research and Treatment of Cancer Quality of Life Questionnaire-core 30, v3 (EORTC QLQ-C30) [35] and the EuroQol Group five dimensions Health 


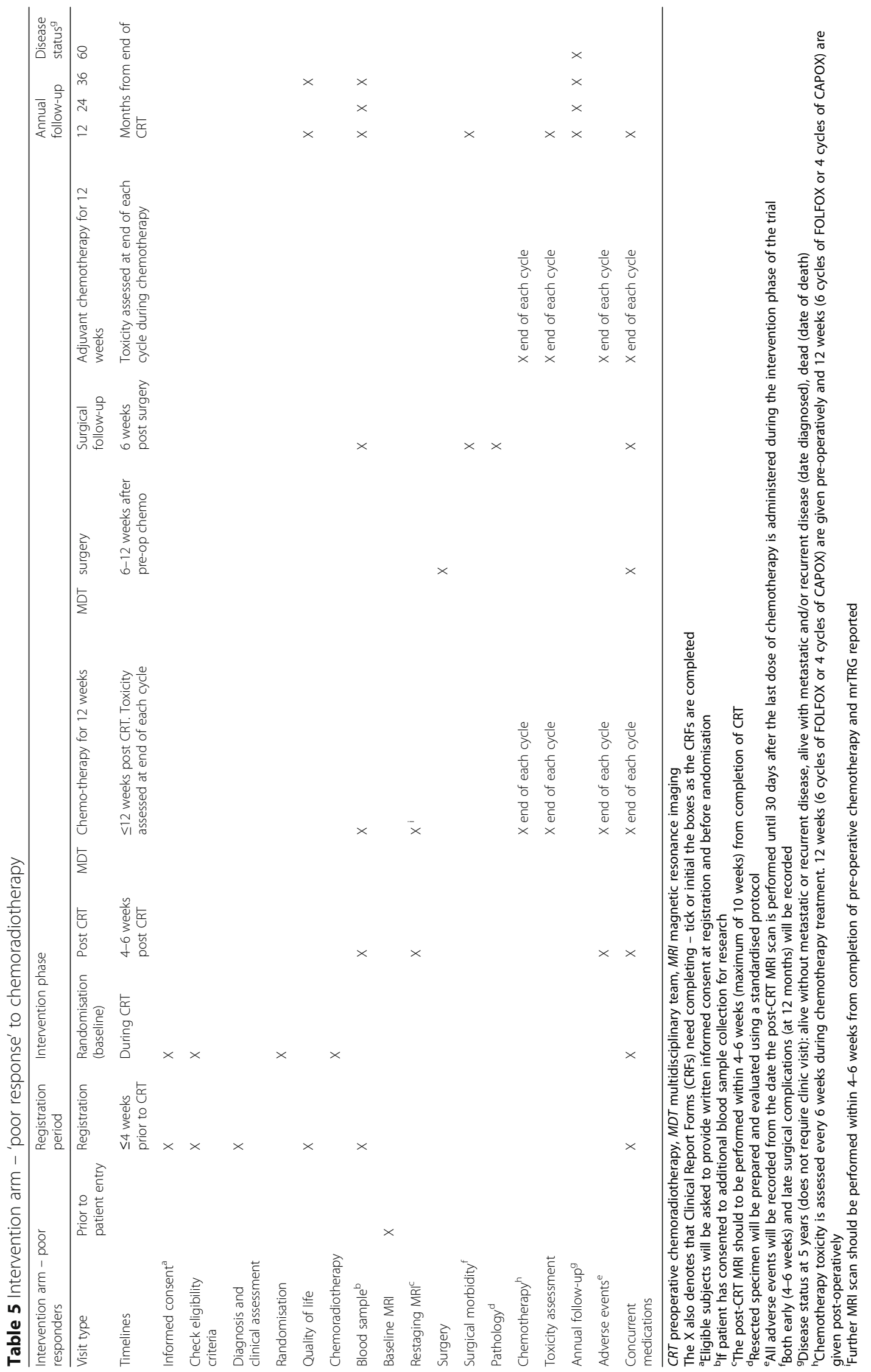


Table 6 SPIRIT diagram for both randomisation arms and intervention arm sub-groups

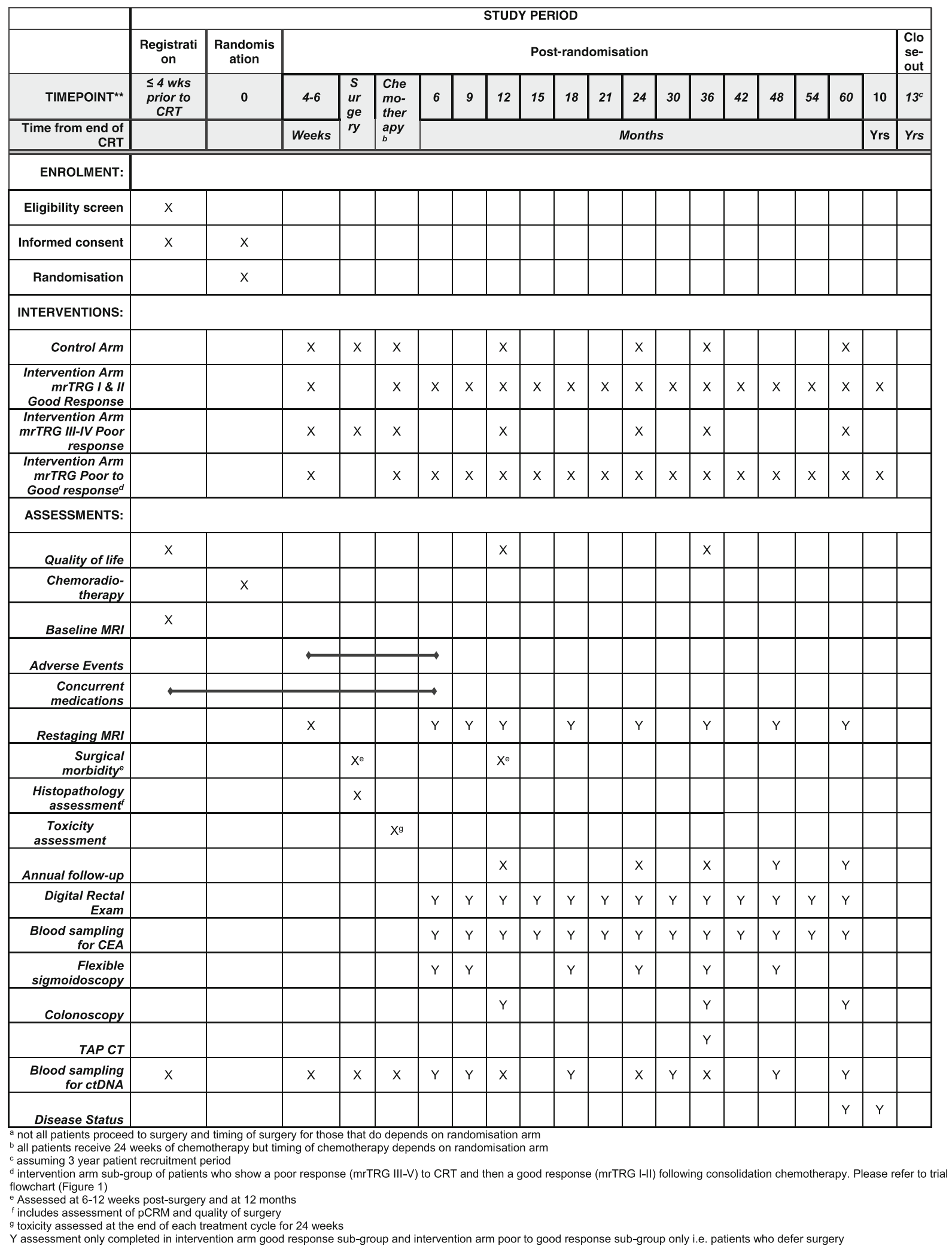


Questionnaire (EQ-5D) [36] to derive a global healthrelated QoL assessment [37]. In addition to this, bowel function will be assessed through the Low Anterior Resection Syndrome (LARS) questionnaire and will be reported according to the severity of bowel dysfunction, as has been reported previously [38].

\section{Withdrawals}

By consenting to the trial, patients will understand that they are consenting to follow-up, data collection, additional MRI scans, additional endoscopy and the collection of biological samples for future research. Selective agreement to these investigations will be treated as a partial withdrawal. Patients have the right to withdraw partially or fully from the study at any time and for any reason without prejudice to their future medical care. Patients' decision for full withdrawal must be recorded in hospital records and a withdrawal CRF should be completed, including primary reason for withdrawal. No further CRFs, except for serious adverse event data, should be completed. Partial withdrawal of consent means that the patient does not wish any further trial treatment but is still willing to provide on-going observational data by continuing with study follow-up. A patient may discontinue trial treatment whenever the treatment is no longer in the patient's best interests, the reasons for doing so should be clearly recorded.

\section{Safety evaluation and reporting of adverse events}

The investigator is responsible for ensuring that all adverse events, adverse reactions, unexpected adverse reactions (UARs), suspected unexpected serious adverse reactions (SUSARs), and serious adverse events (SAEs) observed by the investigator or reported by patients from the date of the post-CRT MRI until 30 days after the last dose of chemotherapy is administered are properly captured in the patients' medical records. Adverse events occurring in all arms of the trial should be reported with the same diligence so that bias is not introduced to the apparent incidence of adverse events observed in either arm. The Trial Management Group (TMG) will review all events and reactions. All SAEs will be reported to the TMG approximately 6-monthly and to the Data Monitoring and Ethics Committee (DMEC) annually.

\section{Discussion}

The current consensus is to plan treatment for rectal cancer using baseline MRI staging [7]. However, this position is changing and some authors believe that there is a moral imperative to inform patients if they have had a cCR following CRT, with a view to offering these patients deferral of surgery [39, 40]. With the accurate detection and surveillance of a cCR still problematic, there is a need to develop validated and reproducible means of assessing response to CRT in rectal cancer in a trial setting. The TRIGGER trial is designed to prospectively evaluate mrTRG as a novel biomarker for assessing CRT response. It is the first randomised control trial in rectal cancer to stratify management according to the grade of response to pre-operative CRT, and thus tailor treatment to achieve optimal oncological and functional outcomes. Initially, the feasibility study will assess the ability to recruit and randomise patients to deliver a mrTRGdirected management. If this is successful, we intend to perform a phase III trial that will assess whether mrTRG can improve QoL and DFS through a personalised dual management approach; 'poor responders' will be offered additional treatment to enable further downstaging and early treatment of systemic relapse risk, and 'good responders' will be offered deferral of surgery, potentially avoiding the morbidity and mortality of surgery.

\section{Trial status}

The TRIGGER trial is open in the feasibility phase, and at the time of submission 20 patients from three centres have been recruited.

\section{Additional files}

Additional file 1: Trial Protocol Version 5.0. (PDF $2102 \mathrm{~kb}$ )

Additional file 2: REGISTRATION Patient Information Sheet Version 1.1 (PDF 180 kb)

Additional file 3: RANDOMISATION Patient Information Sheet Version 4.0. (PDF $410 \mathrm{~kb}$ )

Additional file 4: Completed SPIRIT Guideline Checklist for TRIGGER (DOC $123 \mathrm{~kb}$ )

\section{Abbreviations}

AR: Adverse reactions; CAPOX: Combination chemotherapy treatment with capecitabine with oxaliplatin; cCR: Clinical complete response;

CFS: Colostomy-free survival; CRF: Clinical Report Form; CRM: Circumferential resection margin; CRT: Pre-operative chemoradiotherapy; DFS: Disease-free survival; DMEC: Data Monitoring and Ethics Committee; DR: Distant recurrence; EORTC QLQ-C30: European Organisation for Research and Treatment of Cancer Quality of Life Questionnaire-Core 30, v3; EQ5D: EuroQol Group five dimensions Health Questionnaire;

FOLFOX: Combination chemotherapy treatment with folinic acid, fluorouracil and oxaliplatin modified de Gramont; LARS: Low Anterior Resection Syndrome; MDT: Multidisciplinary team; MHRA: Medicines and Healthcare products Regulatory Agency; MREC: Multicentre Ethical Committee; mrEMVI: MRI-assessed evidence of extramural venous invasion; mrTRG: MRIassessed tumour regression grade; OS: Overall survival; pCR: Pathological complete response; PROM: Patient-reported outcome measure; QoL: Quality of life; SAE: Serious adverse event; SUSAR: Suspected unexpected serious adverse reaction; TRIGGER: Magnetic resonance tumour regression grade as a novel biomarker to stratify management of good and poor responders to chemoradiotherapy: a rectal cancer multicentre randomised control trial; UAR: Unexpected adverse reaction 


\section{Acknowledgements}

Support has been provided through The Institute of Cancer Research and the National Cancer Research Network. PQ and NPW are funded by Yorkshire Cancer Research and supported by the Leeds CRUK Cancer Centre.

\section{Funding}

The TRIGGER trial was developed with the support of a pump-primed grant from The Pelican Cancer Foundation (Ref Battersby_TRIGGER), with ongoing support from The Royal Marsden NHS Foundation Trust and The Institute of Cancer Research LondonNIHR Biomedical Research Centre (Funding Ref A160).

\section{Authors' contributions}

GB, NJB, BJM, SR, DT, RA, NW, PQ, IC, PT, SR and IN participated in the development (sample size calculation, protocol and funding application) of the trial. GB, NJB, BJM, $\mathrm{KT}$ and MF contributed to the implementation (submission to ethics committee, data management, monitoring and patient enrolment). NJB, MD, NW, PQ, KT, GB, $B J M, I C$ and MF helped to draft and to review the paper. NW and PQ wrote the pathology proforma and methodology. RS, DC, RA, SK and NJB wrote the oncology methodology. Translational studies' methodology was written by IC, AS, IN, NV and AM. All authors read and approved the final manuscript.

\section{Ethics approval and consent to participate}

National Research Ethics Committee approval was granted by London Surrey Borders Research Ethics Committee (REC) on 18 December 2015 (15/ LO/1836). The REC issued centralised ethics committee approval in all UK centres. The conduct of the trial has been, and will continue to be, in accordance with the clinical trials regulations, the principles of Good Clinical Practice and the Declaration of Helsinki. A prerequisite to recruiting each patient to trial has been, and will continue to be, signed informed consent to participate.

Version 5.0 of the full trial protocol, including the appendices for the delivery and assessment of all applicable imaging techniques, chemotherapy, radiotherapy, and pathology are available as supporting documents; however, publication of supporting raw data is currently not applicable.

\section{Consent for publication}

Informed written consent was received for publication of the manuscript and figures. Written informed consent was obtained from the patients to participate and for publication of their individual details and accompanying anonymised images in this manuscript. The consent form is held by the Royal Marsden Trials Unit and is available for review by the Editor-in-Chief.

\section{Competing interests}

Professor David Cunningham receives research income from Amgen, AstraZeneca, Bayer, Celgene, Merrimack, Medimmune, Merck Serono and Sanofi. Prof Phil Quirke has pharmaceutical research contracts with Roche/ Ventana and Eisai, and is a member of an advisory board with AMGEN. All other authors have no conflicts of interest.

\section{Publisher's Note}

Springer Nature remains neutral with regard to jurisdictional claims in published maps and institutional affiliations.

\section{Author details}

${ }^{1}$ Pelican Cancer Foundation, The Ark, Basingstoke RG24 9NN, UK. ${ }^{2}$ North Hampshire Hospital Foundation Trust, Basingstoke RG24 9NA, UK. ${ }^{3}$ Department of Medicine Royal Marsden Hospital Sutton, Sutton SM2 5PT, UK. ${ }^{4}$ Velindre Cancer Centre Velindre Hospital Cardiff, Cardiff CF4 7XL, UK. ${ }^{5}$ Gastrointestinal Unit Royal Marsden Hospital Sutton, Sutton SM2 5PT, UK. ${ }^{6}$ Department of Colorectal Surgery, Royal Marsden Hospital London, London SW3 6JJ, UK. ${ }^{7}$ Department of Surgery and Department for Tissue Microarray analysis, University of Southampton, Southampton SO16 6YD, UK. ${ }^{8}$ Pathology and Tumour Biology, Leeds Institute of Cancer and Pathology, Wellcome Trust Brenner Building, St. James's University Hospital, Leeds LS9 7TF, UK. ${ }^{9}$ Department of Pathology Radboud University, Nijmegen 6500HB, Netherlands. ${ }^{10}$ Division of Molecular Pathology Institute of Cancer Research, London SW3 6JB, UK. ${ }^{11}$ Statistics Unit, R\&D Royal Marsden Hospital Sutton, Sutton SM2 5PT, UK. ${ }^{12}$ Department of Radiology, Royal Marsden Hospital Sutton, Sutton SM2 5PT, UK.
Received: 14 December 2016 Accepted: 3 July 2017

Published online: 29 August 2017

\section{References}

1. Rullier E, Denost Q, Vendrely V, Rullier A, Laurent C. Low rectal cancer: classification and standardization of surgery. Dis Colon Rectum. 2013; 56(5):560-7.

2. Habr-Gama A, Perez RO, Nadalin W, et al. Operative versus nonoperative treatment for stage 0 distal rectal cancer following chemoradiation therapy: long-term results. Ann Surg. 2004;240(4):711-7. discussion 7-8.

3. Habr-Gama A, Perez RO. Non-operative management of rectal cancer after neoadjuvant chemoradiation. Br J Surg. 2009;96(2):125-7.

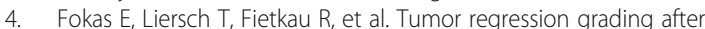
preoperative chemoradiotherapy for locally advanced rectal carcinoma revisited: updated results of the CAO/ARO/AIO-94 Trial. J Clin Oncol. 2014; 32(15):1554-62.

5. Capirci C, Valentini V, Cionini L, et al. Prognostic value of pathologic complete response after neoadjuvant therapy in locally advanced rectal cancer: long-term analysis of 566 ypCR patients. Int J Radiat Oncol Biol Phys. 2008;72(1):99-107.

6. Valentini $\mathrm{V}$, Minsky $\mathrm{BD}$. Tumor regression grading in rectal cancer: is it time to move forward? J Clin Oncol. 2014;32(15):1534-6.

7. The Beyond TME Collaborative. Consensus statement on the multidisciplinary management of patients with recurrent and primary rectal cancer beyond total mesorectal excision planes. Br J Surg. 2013;100(8):E1-E33.

8. Battersby NJ, Moran B, Yu S, Tekkis P, Brown G. MR imaging for rectal cancer: the role in staging the primary and response to neoadjuvant therapy. Expert Rev Gastroenterol Hepatol. 2014;8(6):703-19.

9. Patel UB, Blomqvist LK, Taylor F, et al. MRI after treatment of locally advanced rectal cancer: how to report tumor response-the MERCURY experience. AJR Am J Roentgenol. 2012;199(4):W486-95.

10. Patel UB, Taylor F, Blomqvist L, et al. Magnetic resonance imaging-detected tumor response for locally advanced rectal cancer predicts survival outcomes: MERCURY experience. J Clin Oncol. 2011;29(28):3753-60.

11. Patel UB, Brown $\mathrm{G}$, Rutten $\mathrm{H}$, et al. Comparison of magnetic resonance imaging and histopathological response to chemoradiotherapy in locally advanced rectal cancer. Ann Surg Oncol. 2012;19(9):2842-52.

12. Siddiqui M, Bhoday J, Balyansikova S, Battersby NJ, Chand M, Abulafi AM, Tekkis P, Brown G. Oral posters: inter-observer agreement of radiologists assessing the response of rectal cancers after chemoradiation therapy according to MRI tumour regression grading (mrTRG). Colorectal Dis. 2015;17:11-21.

13. Greenaway K, Hill J, Khatun S, Kurybam A, Meace C, Scott N, Vallance A Van der Meulen J, Walker K, Yelland A, on behalf of ACPGBI. National Bowel Cancer Audit Annual Report. 2015.

14. Scott NHJ, ACPGBI. National Bowel Cancer Audit Progress Report: Tripartite Colorectal Meeting Birmingham. 2014.

15. Emmertsen KJ, Laurberg S, Rectal Cancer Function Study Group. Impact of bowel dysfunction on quality of life after sphincter-preserving resection for rectal cancer. Br J Surg. 2013;100(10):1377-87.

16. Peeters KC, van de Velde CJ, Leer JW, et al. Late side effects of short-course preoperative radiotherapy combined with total mesorectal excision for rectal cancer: increased bowel dysfunction in irradiated patients - a Dutch colorectal cancer group study. J Clin Oncol. 2005;23(25):6199-206.

17. Patient Forum. Low rectal cancer-deferral of surgery? 2014. http:// coloncancersupport.colonclub.com/viewtopic.php?f=1\&t=45894\&sid= ded6e0a46cae3b43f34943d08f97f29c-p333961.

18. Habr-Gama A, Gama-Rodrigues J, Sao Juliao GP, et al. Local recurrence after complete clinical response and watch and wait in rectal cancer after neoadjuvant chemoradiation: impact of salvage therapy on local disease control. Int J Radiat Oncol Biol Phys. 2014;88(4):822-8.

19. Smith JD, Ruby JA, Goodman KA, et al. Nonoperative management of rectal cancer with complete clinical response after neoadjuvant therapy. Ann Surg. 2012;256(6):965-72

20. Habr-Gama A, Sabbaga J, Gama-Rodrigues J, et al. Watch and wait approach following extended neoadjuvant chemoradiation for distal rectal cancer: are we getting closer to anal cancer management? Dis Colon Rectum. 2013;56(10):1109-17.

21. Maas M, Beets-Tan RG, Lambregts DM, et al. Wait-and-see policy for clinical complete responders after chemoradiation for rectal cancer. J Clin Oncol. 2011;29(35):4633-40. 
22. Smith FM, Wiland H, Mace A, Pai RK, Kalady MF. Clinical criteria underestimate complete pathological response in rectal cancer treated with neoadjuvant chemoradiotherapy. Dis Colon Rectum. 2014;57(3): 311-5.

23. Habr-Gama A, Perez RO. The surgical significance of residual mucosal abnormalities in rectal cancer following neoadjuvant chemoradiotherapy ( $\mathrm{Br}$ J Surg. 2012;99: 993-1001). Br J Surg. 2012;99(11):1601. author reply - 2.

24. Smith FM, Chang KH, Sheahan K, Hyland J, O'Connell PR, Winter DC. The surgical significance of residual mucosal abnormalities in rectal cancer following neoadjuvant chemoradiotherapy. Br J Surg. 2012;99(7):993-1001.

25. West $\mathrm{N}$. The surgical significance of residual mucosal abnormalities in rectal cancer following neoadjuvant chemoradiotherapy (Br J Surg. 2012;99: 9931001). Br J Surg. 2012;99(7):1001.

26. Renehan AG, Malcomson L, Emsley R, et al. Watch-and-wait approach versus surgical resection after chemoradiotherapy for patients with rectal cancer (the OnCoRe project): a propensity-score matched cohort analysis. Lancet Oncol. 2016;17(2):174-83.

27. Nielsen LB, Wille-Jorgensen P. National and international guidelines for rectal cancer. Colorectal Dis. 2014;16(11):854-65.

28. NICE. The diagnosis and management of colorectal cancer: NICE clinical guideline 131. 2011. http://www.nice.org.uk/guidance/CG131. Accessed 28 Oct 2014.

29. Garcia-Aguilar J, Smith DD, Avila K, Bergsland EK, Chu P, Krieg RM. Optimal timing of surgery after chemoradiation for advanced rectal cancer: preliminary results of a multicenter, nonrandomized phase II prospective trial. Ann Surg. 2011;254(1):97-102

30. Glynne-Jones R, Sebag-Montefiore D, Maughan TS, Falk SJ, McDonald AC. A phase I dose escalation study of continuous oral capecitabine in combination with oxaliplatin and pelvic radiation (XELOX-RT) in patients with locally advanced rectal cancer. Ann Oncol. 2006;17(1):50-6.

31. Rodel C, Liersch T, Hermann RM, et al. Multicenter phase II trial of chemoradiation with oxaliplatin for rectal cancer. J Clin Oncol. 2007;25(1):110-7.

32. Rodel $\mathrm{C}$, Liersch $\mathrm{T}$, Becker $\mathrm{H}$, et al. Preoperative chemoradiotherapy and postoperative chemotherapy with fluorouracil and oxaliplatin versus fluorouracil alone in locally advanced rectal cancer: initial results of the German CAO/ARO/AIO-04 randomised phase 3 trial. Lancet Oncol. 2012;13(7):679-87.

33. Battersby NJ, How P, Moran B, et al. Prospective validation of a low rectal cancer magnetic resonance imaging staging system and development of a local recurrence risk stratification model: The MERCURY II Study. Ann Surg. 2016;263(4):751-60.

34. Sadanandam A, Lyssiotis CA, Homicsko K, et al. A colorectal cancer classification system that associates cellular phenotype and responses to therapy. Nat Med. 2013;19(5):619-25.

35. Aaronson NK, Ahmedzai S, Bergman B, et al. The European Organization for Research and Treatment of Cancer QLQ-C30: a quality-of-life instrument for use in international clinical trials in oncology. J Natl Cancer Inst. 1993;85(5):365-76

36. Sharma A, Sharp DM, Walker LG, Monson JR. Predictors of early postoperative quality of life after elective resection for colorectal cancer. Ann Surg Oncol. 2007;14(12):3435-42.

37. Fayers P, Aaronson N, Bjordal K, et al. The EORTC QLQ-C30 Scoring Manual (3rd Edition), Brussels European Organisation for Research and Treatment of Cancer. 2001.

38. Emmertsen KJ, Laurberg S. Low anterior resection syndrome score: development and validation of a symptom-based scoring system for bowel dysfunction after low anterior resection for rectal cancer. Ann Surg. 2012;255(5):922-8.

39. Heald RJ, Beets G, Carvalho C. Report from a consensus meeting: response to chemoradiotherapy in rectal cancer-predictor of cure and a crucial new choice for the patient: on behalf of the Champalimaud 2014 Faculty for 'Rectal cancer: when NOT to operate'. Colorectal Dis. 2014;16(5):334-7.

40. São Julião GP, Smith FM, Macklin CP, George ML, Wynn GR. Opinions have changed on the management of rectal cancer with a complete clinical response to neoadjuvant chemoradiotherapy. Colorectal Dis. 2014:16(5):392-4.

\section{Submit your next manuscript to BioMed Central and we will help you at every step:}

- We accept pre-submission inquiries

- Our selector tool helps you to find the most relevant journal

- We provide round the clock customer support

- Convenient online submission

- Thorough peer review

- Inclusion in PubMed and all major indexing services

- Maximum visibility for your research

Submit your manuscript at www.biomedcentral.com/submit 